\title{
CORRIGENDUM
}

\section{Universality in network dynamics}

Baruch Barzel and Albert-László Barabási

Nature Physics 9, 673-681 (2013); published online 8 September 2013; corrected after print 9 October 2013.

In the version of this Article originally published, the expression for $G_{i j}$ on page 673 should have included an absolute value sign. In addition, the caption for Fig. 1b1-c4 was missing the final wording: (note that the bounded distributions here and throughout are normalized to have mean zero and variance one). These errors have been corrected in the HTML and PDF versions of the Article. 